\section{Kanton Solothurn: «Der Widerspenstigen Zähmung ...»}

\section{P. Eisenhut}

\section{Stein des Anstosses: \\ Der sogenannte "Scheckmissbrauch"}

Gemäss Art. 42 des geltenden Krankenversicherungsgesetzes (KVG) schulden die Versicherten den Leistungserbringern (Ärzteschaft, Apotheker etc.) die Vergütung der Leistung, wenn Versicherer und Leistungserbringer nichts anderes vereinbart haben. Die Versicherten haben in diesem Fall gegenüber dem Versicherer einen Anspruch auf Rückerstattung (System des "tiers garant»). Seither haben sich auch im Kanton Solothurn die Fälle gehäuft, in denen Patienten die Arztrechnungen nicht bezahlten, obwohl ihnen ihr Krankenversicherer den Betrag ausbezahlt hat. Solche Fälle des "Scheckmissbrauchs" stellten nach Auffassung der solothurnischen Verwaltungsbehörden keine Zahlungsunfähigkeit, sondern lediglich mangelnder Zahlungswille dar.

Auf Beschwerde eines Arztes entschied das Verwaltungsgericht des Kantons Solothurn am 22. April 1996, das Sanitätsgesetz stelle einzig auf die Zahlungsunfähigkeit des Patienten ab. Diese sei zu vermuten, wenn ein Patient fruchtlos gepfändet und gegen ihn ein Verlustschein ausgestellt werde. Dadurch seien die Voraussetzungen, welche das Sanitätsgesetz an die Zahlungspflicht des Gemeinwesens knüpfe, erfüllt, weshalb das Verwaltungsgericht die betroffene Gemeinde anwies, das Arzthonorar zu bezahlen. In einem weiteren Urteil vom 15. Oktober 1996 entschied das Verwaltungsgericht, dass die Gemeinwesen auch die Verlustscheinkosten zu übernehmen haben. Den vom Verwaltungsgericht beurteilten Sachverhalten lagen Notfallbehandlungen zugrunde, was das Departement dazu veranlasste, in seinen Richtlinien die verwaltungsgerichtliche Rechtsprechung auf Notfallbehandlungen zu beschränken. In einem weiteren Urteil vom 5. November 1997 entschied das Verwaltungsgericht schliesslich, seine Rechtsprechung gelte auch für Nichtnotfallbehandlungen.

So ergab sich, dass Ärzte - egal, ob ein Scheckmissbrauch vorlag oder nicht - unbezahlte Arzthonorare bei nachgewiesener Zahlungsunfähigkeit des Schuldners bei der Sozialbehörde an dessen Wohnsitz geltend machen konnten. Diese Praxis hat sich bei allen Beteiligten gut eingespielt und funktionierte während annähernd $1 \frac{1}{2}$ Jahren reibungslos.

\section{Der Versuch, das neue Gesundheitsgesetz vorwirken zu lassen ...}

Parallel zur Entwicklung der oben skizzierten Rechtsprechung befasste sich der Regierungsrat des Kantons Solothurn mit einem Entwurf für ein neues Gesundheitsgesetz, welches das veraltete Sanitätsgesetz ablösen sollte. Das Kantonsparlament begann mit der Beratung des Gesetzesentwurfs Ende Dezember 1998.
Patrik Eisenhut

c/o Schwegler \& Partner

Rechtsanwälte Notariat

Effingerstrasse 8

$\mathrm{CH}-3001$ Bern \begin{abstract}
dem zu Jahresbeginn in Kraft getretenen Ges heitsgesetz erläutert.
\end{abstract}


Das inzwischen per 1. Januar 2000 in Kraft gesetzte Gesundheitsgesetz auferlegt Ärzten, Zahnärzten und Chiropraktoren in § 25 ebenfalls eine Behandlungspflicht. Im Gegensatz zum früheren Gesetz wurde diese allerdings auf ärztliche Behandlungen von Sozialhilfe empfangenden Personen beschränkt. Die Vorlage wurde in diesem Punkt im Kantonsrat unwidersprochen und unverändert angenommen und am 27. Januar 1999 mit einer komfortablen Mehrheit verabschiedet. Dieses unter demokratischen Gesichtspunkten einwandfreie Vorgehen wurde leider von den Vertretern der Einwohnergemeinden gar nicht erst abgewartet.

Mit einer Motion vom 17. Februar 1998 verlangte Kantonsrat und Gemeindepräsident Kurt Zimmerli, Oensingen, vom Regierungsrat, die Sanitätsverordnung sei dahingehend abzuändern, dass der Vollzug des Inkassowesens (durch die Ärzteschaft) die Gemeinden nicht mehr belasten könne. In seiner Stellungnahme zur Motion erachtete der Regierungsrat des Kantons Solothurn eine Anpassung an die veränderten Verhältnisse in dem Sinne als möglich, dass die Gemeinden subsidiär nur noch für ausstehende Zahlungen vom Unterstützungsbedürftigen (Empfängern von Sozialhilfe) hafteten, entsprechend der neuen Formulierung im Entwurf des Gesundheitsgesetzes. Der Regierungsrat versuchte mit anderen Worten auf dem Weg einer Verordnungsänderung den vom Verwaltungsgericht in drei Urteilen unzweideutig ausgelegten Gesetzeswortlaut zurechtzubiegen, so dass die Honorargarantie im wesentlichen entfiel. Dass solches gegen fundamentale Grundsätze des allgemeinen Verwaltungsrechts verstösst, hätte mindestens den Verwaltungsjuristen klar sein müssen.

Kantonsrat Peter Meier erhob dagegen mit weiteren 35 Unterzeichnern ein Veto. Der Regierungsrat beharrte in seiner Stellungnahme zum Veto auf der Gesetzeskonformität der Verordnungsänderung. Begründet wurde dies mit den gleichen Argumenten, mit denen das Departement des Innern wenige Jahre zuvor vor Verwaltungsgericht dreimal gescheitert war. Schliesslich polemisierte der Regierungsrat in seiner Stellungnahme auch gegen die Ärzte, denen er vorwarf, sie hätten die Situation des gehäuften Scheckmissbrauchs selbst verursacht, in dem sie mit den Krankenversicherern das System des "tiers garant" vereinbart hätten. Dabei wurde ausgeblendet, dass dieses Problem in der ganzen Schweiz akut ist (vgl. dazu die Debatten im Bundesparlament im Zusammenhang mit der ersten Teilrevision des KVG) und das System des "tiers garant» nicht einseitig von den Ärzten gewählt wurde, sondern im Gesetz als Grundsatz statuiert ist, von dem lediglich durch vertragliche Vereinbarung mit den Krankenversichern abgewichen werden kann. Das Veto gegen die Änderung der Sanitätsverordnung wurde am 16. Dezember 1998 abgelehnt und die Verordnungsänderung drei Tage später ohne Übergangsbestimmung in Kraft gesetzt.

Die offensichtliche Gesetzwidrigkeit der Verordnungsänderung veranlasste die FMH Inkasso Services AG beim Departement des Innern des Kantons Solothurn eine Musterbeschwerde zu erheben. Der am
23. August 1999 ergangene Musterentscheid hielt stur an der angeblichen Gesetzeskonformität der Verordnungsänderung fest. Lediglich das Fehlen von Übergangsbestimmungen schien dem Departement problematisch, weshalb es in einer neuen Richtlinie die Einwohnergemeinden anwies, es seien künftig die Arztkosten für Behandlungszeiträume vor dem 19. Dezember 1998 weiterhin durch das zuständige Gemeinwesen zu übernehmen. Die FMH Inkasso Services AG liess sich durch diesen Teilerfolg in ihrer Auffassung nicht beirren und veranlasste eine neue Musterbeschwerde.

In einem Entscheid vom 19. Juli 2000 hat das Verwaltungsgericht dem Departement des Innern seine bereits in drei Entscheiden ausgeführten Überlegungen mit grosser Geduld erneut zur Kenntnis gebracht und festgestellt, der abgeänderte Verordnungswortlaut sei durch das Sanitätsgesetz nicht gedeckt, also nicht gesetzeskonform. Damit ist endgültig geklärt, dass bezüglich der Kosten für Arztbehandlungen, welche unter der Geltung des alten Sanitätsgesetzes (bis 31. Dezember 1999) stattgefunden haben, die Einwohnergemeinden haften, soweit die Patienten als Schuldner der Honorarforderung zahlungsunfähig im Sinne des Sanitätsgesetzes sind. Das Departement des Innern hat sich diesem Verdikt gebeugt und die Einwohnergemeinden, deren Verwirrung heute grösser denn je ist, mit einer neuen Richtlinie entsprechend angewiesen.

\section{Die Rechtslage im Kanton Solothurn für Behandlungszeiträume ab 1. Januar 2000}

Das mit Inkrafttreten des Gesundheitsgesetzes (GG) geltende, neue System ergibt sich aus nachfolgender Tabelle, welche der Sozialhilfe-Information Nr. 3/1999 des Amtes für Gemeinden und soziale Sicherheit des Kantons Solothurn entnommen wurde.

\section{Normalfall (linke Spalte)}

Das Gesundheitsgesetz sieht keine Übernahme von nicht erhältlichen Arzthonoraren durch das Gemeinwesen mehr vor.

\section{Ausnahmefall (rechte Spalte)}

Bei Sozialhilfe empfangenden Personen werden nicht bezahlte Arztrechnungen zu den obenerwähnten Konditionen dann übernommen, wenn innert sechs Monaten nach Behandlungsbeginn dem Patienten eingeschrieben die Betreibung angedroht wurde. Dies stellt im Vergleich zur bisherigen Praxis eine Erleichterung dar, weil ausdrücklich auf die Betreibung bis zum Verlustschein verzichtet wird. Anderseits zwingt dieses System die Rechnung stellenden Ärztinnen und Ärzte dazu, den von ihnen erbrachten Behandlungsaufwand zügig abzurechnen und ihre Debitoren effizient $\mathrm{zu}$ bewirtschaften. Um ihren Honoraranspruch gegenüber dem Staat nicht zu verwirken, muss spätestens zwei Monate nach Behandlungsbeginn abgerechnet und den Patienten die Rechnung zugestellt werden. Bei unbezahlten Rech- 
nungen ist eine erste Mahnung spätestens 40 Tage nach Rechnungsstellung zu versenden. Weitere Mahnungen sind aus rechtlicher Optik grundsätzlich nicht notwendig. Ziehen Sie es vor, eine zweite Mahnung zu versenden, so sollte diese nach spätestens 14 Tagen erfolgen. Wird auch auf eine zweite Mahnung nicht reagiert, wäre umgehend eingeschrieben die Betreibung anzudrohen. Der skizzierte Zeitplan ermöglicht Ihnen, Ihren Obliegenheiten innerhalb der von der Verwaltung festgelegten Frist von sechs Monaten nachzukommen. Die Einhaltung dieser sechsmonatigen Verwirkungsfrist liegt folglich in der Verantwortung der Ärzteschaft. Insbesondere bei der Vergabe von Inkassoaufträgen ist diesem Aspekt die nötige Aufmerksamkeit zu schenken.

\section{Ausnahmefall (mittlere Spalte)}

Unabhängig davon, ob jemand Sozialhilfe bezieht, kann der Honoraraufwand für Notfalldienstbehandlungen der zuständigen Einwohnergemeinde $\mathrm{zu}$ $100 \%$ in Rechnung gestellt werden, wenn innert sechs Monaten ab Notfalldienstbehandlung mittels Einschreiben die Betreibung angedroht wurde. Dabei ist $\mathrm{zu}$ beachten, dass Notfallbehandlungen in der Arztpraxis nicht unter diese Regelung, sondern unter den Normalfall in der ersten Spalte fallen. Auch Nachbehandlungen von Notfalldienstpatienten in der eigenen Arztpraxis fallen nicht unter die Ausnahme in der mittleren Spalte.

\section{Schlussfolgerungen}

Neuerdings trägt die Ärzteschaft im Kanton Solothurn unter Vorbehalt der vorstehend erwähnten Ausnahmen das Debitorenrisiko selbst. Dieses kann durch geeignete Massnahmen zwar beschränkt, jedoch nie gänzlich ausgeschlossen werden. Ob Patienten im Zeitpunkt des Behandlungsbeginnes Sozialhilfe beziehen, wird in der Regel durch den behandelnden Arzt nicht leicht festzustellen sein. Eine Rückfrage bei der Einwohnergemeinde ist denkbar, wobei damit zu rechnen ist, dass sich diese auf die Datenschutzgesetzgebung beruft. Womöglich kann aus dem Gespräch mit den Patienten geschlossen werden, ob sie Sozialhilfe empfangen. Die Wahrung des Honoraranspruchs gegenüber dem Gemeinwesen setzt ein effizientes Abrechnungs- und Mahnwesen voraus. Bei längerem Zuwarten droht Anspruchsverlust.

\section{Tabelle 1}

Ambulante ärztliche Tätigkeit.

\begin{tabular}{|c|c|c|}
\hline $\begin{array}{l}\text { Beistandspflicht des Arztes } \\
\text { Hippokratischer Eid, Standesregeln; } \\
\text { § } 24 \text { Abs. } 1 \text { GG }\end{array}$ & $\begin{array}{l}\text { Teilnahmepflicht des Arztes } \\
\S 24 \text { Abs. } 2 \text { GG }\end{array}$ & $\begin{array}{l}\text { Behandlungspflicht des Arztes } \\
\S 25 \text { GG }\end{array}$ \\
\hline $\begin{array}{l}\text { Keine Übernahme unerhältlicher Arzt- } \\
\text { rechnungen durch das Gemeinwesen } \\
\text { (ausgenommen Sozialhilfeempfänger } \\
\text { und Sozialhilfeempfängerinnen, } \\
\text { vgl. 3. Spalte). }\end{array}$ & $\begin{array}{l}\text { Übernahme unerhältlicher Arzt- } \\
\text { rechnungen zu } 100 \% \text {, wenn innert } \\
6 \text { Monaten ab Notfalldienstbehandlung } \\
\text { (Beleg: Arztrechnung) eingeschrieben } \\
\text { die Betreibung angedroht wurde } \\
\text { (gilt für alle Patientinnen und Patienten). } \\
\text { Zuständige Gemeinde übernimmt } \\
\text { ausstehende Arztrechnungen aus Notfall- } \\
\text { dienst zu } 100 \% \text {. }\end{array}$ & $\begin{array}{l}\text { Übernahme unerhältlicher Arzt- } \\
\text { rechnungen zu 75\%, wenn innert } \\
6 \text { Monaten nach Behandlungsbeginn } \\
\text { (Beleg: Arztrechnung) eingeschrieben } \\
\text { die Betreibung angedroht wurde. } \\
\text { Zuständige Gemeinde übernimmt } \\
\text { ausstehende Arztrechnungen mit einer } \\
\text { Ermässigung von 25\%, Arzneimittel } \\
\text { ausgenommen. }\end{array}$ \\
\hline
\end{tabular}

